\title{
Contributions de la linguistique interactionnelle
}

\author{
Lorenza Mondada \\ Laboratoire ICAR, CNRS \& Université de Lyon \\ Département Sciences du Langage, Université Lyon2
}

\section{Introduction}

Ce texte a pour objectif d'expliciter un certain nombre d'enjeux qui se posent de manière générale en sciences du langage aujourd'hui et qui reçoivent une réponse spécifique de la part de la linguistique interactionnelle. Cela permet de situer la contribution d'une littérature récente et souvent encore peu connue en France et de poser les bases pour une discussion sur ses évolutions actuelles.

Une des exigences de plus en plus affirmées au sein de la linguistique contemporaine concerne la nécessité d'articuler différents paliers d'analyse, qui s'exprime de diverses manières selon les paradigmes théoriques : articulations entre syntaxe et pragmatique, entre propriétés structurelles de la langue et ses usages en société, entre contraintes formelles et fonctionnelles. Plusieurs courants linguistiques ont joué un rôle fondamental dans le développement de ces interfaces: on peut mentionner la linguistique fonctionnelle de Talmy Givon, les développements récents de la construction grammar, les différentes formes d'émergentisme, l'étude de la grammaticalisation qui prend de plus en plus en compte les contraintes pragmatique et discursives, voire interactionnelles.

Cette première exigence va de pair avec une seconde, consistant à reconnaitre l'importance de travailler sur des corpus de données attestées : cette exigence, critique aussi bien envers la démarche introspective qu'envers les techniques d'élicitation de réponses contraintes sur le langage, répond à l'enjeu théorique de développer des usage-based grammars.

La linguistique interactionnelle est un paradigme récent, qui a émergé comme tel durant les années 90 , tout en reposant sur les acquis de l'analyse conversationnelle, apparue dans les années 60 . Elle répond de manière spécifique à ces exigences, en développant un projet systématique d'étude de la langue dans l'interaction, sur la base d'enregistrements d'interactions en situation naturelle. Très présente dans la linguistique scandinave, anglo-saxonne et allemande, elle l'est encore peu dans le paysage de la linguistique française, tout en commençant à s'y développer, comme en témoignent les articles présents dans cette section. Dans ce qui suit nous allons rappeler quelques repères historiques (1.), préciser la démarche relative aux corpus (2.) et à leur analyse (3.), avant d'expliciter la manière dont s'imbriquent analyse de l'organisation de l'interaction et analyse des ressources linguistiques (4.), avant de conclure sur quelques enjeux majeurs qui ouvrent sur un chantier encore largement à défricher (5.).

\section{Quelques repères historiques}

Il est intéressant de constater que les principes de l'interactionnisme émergent chez des auteurs très disparates au début du XXe siècle - aussi divers que les pragmatistes américains, tels que Mead, Dewey, ou James, et les auteurs soviétiques tels que Bakhine-Volosinov et Vygotsky. Tous reconnaissent de différentes manières l'ancrage des dynamiques de l'esprit comme du langage dans la pratique et plus précisément dans les pratiques interactionnelles.

Pour ne prendre qu'un exemple, Volosinov affirme dès 1929 que :

Word is a two-sided act. It is determined equally by whose word it is and for whom it is meant. As word, it precisely the product of the reciprocal relationship between speaker and listener, addresser and addressee [...] A word is territory shared by both addresser and addressee, by the speaker and his interlocutor. (Volosinov, 1929, trad. anglaise, $1973: 86$ ). 
Cette reconnaissance précoce du rôle de l'interaction dans la structuration du langage et de l'action réciproque des co-participants non seulement sur la compréhension de ce qui est échangé mais sur la production même (cf. Bronckart et Brès, ici-même, pour une discussion de cet héritage) entre en résonance avec les contributions plus récents de la linguistique interactionnelle - notamment le travail pionnier de Goodwin, dans son analyse, dès 1979, de l'énoncé comme étant co-produit interactionnellement par les interlocuteurs dans les détails les plus subtils de son énonciation (voir Goodwin, 1981), permettant d'inscrire le principe interactionnel au sein d'unités traditionnellement traitées comme relevant typiquement de l'activité monologale du locuteur ${ }^{1}$.

C'est dans les années 60 que l'héritage de l'interactionnisme dans différentes disciplines (sociologie, psychologie, linguistique) donne lieu à la formulation de modèles d'analyse qui joueront un rôle fondamental pour le développement de la pragmatique, de l'analyse du discours et de l'analyse de l'interaction en linguistique, avec de fortes ramifications interdisciplinaires. Goffman y intervient comme un point de référence central, inspirant aussi bien l'analyse conversationnelle (Sacks, Schegloff, Jefferson, 1979, 1977, Schegloff \& Sacks, 1973 ; Sacks, 1992 ; Schegloff, 2007 - cf. Lerner 2004 pour une anthologie d'écrits des fondateurs), sur le modèle de l'Ecole de Birmingham (Sinclair \& Coulthard, 1975), qui sera repris en France par l'Ecole de Genève (Roulet, 1980, Roulet et alii 1985), sur la sociolinguistique interactionnelle (Gumperz, 1982), sur les théories de la politesse (Brown \& Levinson, 1978) reprises en France par les travaux de Kerbrat-Orecchioni (1990-94).

Dans ce contexte, l'analyse conversationnelle (AC) apparaît dans les années 60 comme une manière de développer le programme ethnométhodologique (fondé par Garfinkel, 1967) en ancrant l'étude de l'organisation située des pratiques sociales dans l'analyse fine d'enregistrements - audio à l'époque - de pratiques interactionnelles naturelles. L'analyse conversationnelle est le premier courant qui a répondu de manière systématique à l'exigence de travailler sur des données enregistrées naturelles : l'intérêt pour la parole conversationnelle est à maintes reprises signalé par Sacks (1984) comme ne relevant pas d'un intérêt particulier pour le langage en soi mais comme étant un byproduct de l'exigence de fonder la description des «méthodes » par lesquelles les co-participants organisent, interprètent et produisent leurs contributions à l'interaction sur des enregistrements de situations de parole naturelles. Reste que cette exigence produira un corps important d'études sur les caractéristiques systématiques de la parole-eninteraction, centrées sur la conversation d'abord - entendue comme l'usage prototypique du langage le moins contraint et donc offrant la plus grande diversité de formats séquentiels - sur des interactions institutionnelles et professionnelles ensuite, permettant d'observer la spécialisation des formats conversationnels dans divers contextes sociaux (Drew \& Heritage, 1982).

Le but de l'AC, dans sa généralité, est de rendre compte de la conversation comme un phénomène ordonnné. Cet ordre toutefois n'est pas préexistant à la conversation, ni n'obéit à des principes qui lui seraient extérieurs et qui ne feraient qu'y être reproduits : cet ordre est produit in situ, par les participants, de manière endogène. L'objet de l'analyse est donc moins l'ordre lui-même que la manière dont il est accompli, grâce à des procédés - des méthodes - mis en oeuvre par les participants, qui sont, eux, reproductibles et généralisables. Ainsi que le résume Psathas, la tâche de l'analyste est par conséquent de produire une description des principes d'ordre, tels que traités par les participants :

Conversation analysis studies the order/organization/orderliness of social action, particularly those social actions that are located in everyday interaction, in discursive practices, in the sayings/tellings/doings of members of society.

Its basic assumptions are:

1. Order is a produced orderliness.

2. Order is produced by the parties in situ; that is, it is situated and occasioned.

3. The parties orient to that order themselves; that is, this order is not an analyst's conception, not the result of the use of some preformed or preformulated theoretical conceptions concerning what action should/must/ought to be, or based on generalizing or summarizing statements about what action generally/frequently/often is.

4. Order is repeatable and recurrent.

5. The discovery, description, and analysis of that produced orderliness is the task of the analyst. 


\begin{abstract}
6. Issues of how frequently, how widely, or how often particular phenomena occur are to be set aside in the interest of discovering, describing, and analyzing the structures, the machinery, the organized practices, the formal procedures, the ways in which order is produced.

7. Structures of social action, once so discerned, can be described in formal, that is, structural, organizational, logical, atopically contentless, consistent, and abstract, terms.

(Psathas, 1995, 2-3)
\end{abstract}

Fidèle en cela à son inspiration ethnométhodologique, l'analyse conversationnelle observe un double principe : d'une part, elle reconnaît que l'action est située, occasionnée, sensible aux contingences et orientée vers le contexte de sa production et vers sa spécificité : elle est irrémédiablement indexicale. D'autre part part, elle considère que cette action est localement organisée grâce à des procédés qui, eux, traversent les contextes de leur mise en oeuvre et se caractérisent non seulement par leur récurrence mais plus fondamentalement par leur systématicité. La prise en compte de ces deux aspects ensemble - alors même qu'ils sont souvent traités comme contradictoires ou mutuellement exclusifs - est fondamentale pour apprécier le programme de l'AC.

La linguistique interactionnelle apparaît dans ce contexte, en réponse à la question de savoir quel rôle, à la fois structurant et structuré, jouent les ressources grammaticales dans et pour l'organisation de l'interaction. Dans l'émergence de ce courant, la publication de l'ouvrage collectif Grammar in Interaction, édité par Ochs, Schegloff \& Thomspon (1996), fonctionne comme un déclencheur, avec l'ambition de revisiter la grammaire à l'aune de l'interaction. Le terme de « linguistique interactionnelle » apparaît quelques années plus tard (Mondada, 1998/2001; Selting \& Couper-Kuhlen, 2001a, 2001b) ${ }^{2}$. Depuis, il a eu une fortune considérable, surtout en Europe, en parvenant à unifier un nombre croissant de chercheurs, autour d'un congrès régulier (International Conference on Conversation Analysis, ICCA, Copenhagen, 2002, Helsinki, 2006, Mannheim, 2010) et avec des ouvrages collectifs de référence (Ochs, Schegloff, Thompson, 1996 ; Couper-Kuhlen \& Selting, 2001 ; Ford, Fox, \& Thompson, 2002, Hakulinen \& Selting, 2005). L'objectif qui est poursuivi est double (cf. infra 5.) : décrire la manière dont les structures formelles de la grammaire - entendues comme des ressources exploitées par les participants sont mobilisées de manière à la fois adéquate à et contrainte par l'organisation de l'interaction et, en retour, montrer comment l'organisation de l'interaction laisse émerger des formes et des emplois formels particuliers, répondant à des fonctionnalités interactionnelles.

La situation dans le domaine francophone est paradoxale: alors que les travaux s'intéressant à l'interaction y apparaissent dans les années 80 , certains faisant une plus ou moins grande place à l'analyse conversationnelle et se diffusent largement dans les années 90 (voir les travaux de Kerbrat-Orecchioni, 1990-94), les travaux d'analyse conversationnelle stricto sensu y sont moins nombreux ${ }^{3}$. Aujourd'hui la production en linguistique interactionnelle est encore largement moins développée à côté d'autres langues comme l'anglais, les langues scandinaves, le finnois, l'allemand ou le japonais.

\title{
3 L'importance des corpus de données enregistrées en contexte naturel
}

Malgré la reconnaissance, dès Saussure, de la langue parlée comme objet premier de la linguistique, les normes de l'écrit on continué pendant longtemps à configurer les modèles du langage et de la langue (Harris, 1981, Linell, 2005). Même si les technologies pour enregistrer la parole vive sont disponibles depuis très longtemps - il suffit de penser à la création du Phonogrammarchiv de Vienne en 1899, de celui de Berlin en 1902 et des Archives de la parole par Ferdinant Brunot en 1911; mais aussi, en anthropologie, au fait que Haddon filme des danses et cérémonies à Torres Strait dès 1898 - ce n'est que à partir des années 50 que ces techniques commencent à être systématiquement utilisées par certains chercheurs. La Natural History of an Interview (McQuown, 1971), basée sur un entretien entre Bateson et Doris, une patiente, et filmé en 1955, joue un rôle pionnier non seulement en tant que corpus enregistré mais aussi pour l'exploitation systématique et interdisciplinaire qui en a été faite par ses auteurs - dont la tentative de codage des mouvements du corps par Birdwhistell est peut-être le plus connue (voir Mondada, 2006a, note 2, 315-316). 
Depuis les années 60, l'AC est le mouvement qui a le plus thématisé l'importance des données enregistrées « naturelles », condition sine qua non d'une étude détaillée de l'interaction telle qu'elle est localement organisée par les participants (Sacks, 1984). Sacks s'est intéressé très tôt aux possibilités d'utiliser non seulement le magnétophone mais aussi la caméra (voir l'article de Sacks \& Schegloff, 1971, publié en 2002). Mais c'est Charles Goodwin, fort d'une formation professionnelle dans la production filmique, qui dès les années 70 réalise un grand nombre de corpus vidéo (analysés dans sa thèse, publiée en 1981, mais aussi largement diffusés dans la communauté conversationnaliste).

L'importance que prennent les moyens d'enregistrement et par là les exigences en matière de corpus (de « naturally occurring data ») va de pair avec une approche du langage comme pratique sociale : la langue, aux antipodes d'un système abstrait, y est conçue comme un ensemble de ressources indexicalement liées aux conditions de leur usage, prenant sens dans l'action, et donc fortement liée à sa temporalité émergente (cf. Mondada, 2005, 2006b).

Plus précisément, la linguistique interactionnelle inspirée de l'AC insiste tout particulièrement sur l'importance d'observer les activités des participants dans des événements de la vie sociale ordinaire, dans des naturally occurring interactions, c'est-à-dire des interactions qui auraient eu lieu même en l'absence du chercheur et qui n'ont pas été élicitées ou orchestrées par lui en vue de leur enregistrement. Cette exigence est articulée de manière cohérente avec les exigences de l'analyse. Une de ses raisons fondamentales relève du caractère à la fois context-free, context-shaped et context-renewing des pratiques des locuteurs: d'une part, celles-ci s'organisent de manière localement située, au sens où elles sont dotées d'une indexicalité inévitable et généralisée, en s'ajustant aux contingences affectant les événements et les activités - tout en contribuant réflexivement à en (re)définir le contexte. Transposer ces pratiques dans un autre contexte signifierait les altérer de manière radicale, puisqu'elles s'ajusteraient à d'autres contingences.

Ce qui constitue les données a ainsi profondément changé durant ces dernières décennies en linguistique : on est passé des exemples notés à la volée, sans aucun enregistrement et donc sans aucune garantie quant à la précision de la notation, à des enregistrements qu'on a eu tendance, pendant un certain temps, à délaisser dès qu'était établie leur transcription, ainsi autonomisée, fondant l'analyse de chercheurs qui ne revenaient pas toujours aux bandes originales; à des transcriptions alignant le texte avec sa source et permettant de matérialiser grâce à la technologie (grâce à des logiciels tels que CLAN, ELAN, Praat, ANVIL...) l'indissociabilité entre données primaires et données secondaires, garantie d'un travail effectué en référence constante aux données enregistrées.

L'AC est un des courants qui a massivement contribué à attirer l'attention vers l'importance de la transcription soigneuse des données, non seulement du point de vue de la représentation du segmental, mais aussi de la temporalité de son déroulement pas à pas (chevauchements, pauses, accélérations, décélérations, structures rythmiques). Celle-ci concerne aussi bien la parole que les autres ressources multimodales (gestes, regards, mimiques faciales, mouvements du corps, manipulations d'artefacts) qui pose des problèmes complexes de représentation détaillée de la coordination et synchronisation fine entre les participants (voir sur la transcription de données interactionnelles en français Bonu, 2002, Bürki \& De Stefani, 2006, Mondada, 2000, 2006a, 2007a).

Cette attention pour les données s'incarne depuis les années 60 dans une pratique d'analyse qui est indissociable de la pratique de la transcription et de l'enregistrement des données : elle répond à l'exigence de travailler sur des données dont on a une connaissance approfondie. Néanmoins, cela n'exclut pas que pour une systématisation des analyses (cf. infra), de grandes masses de données soient nécessaires, mises à disposition par des échanges informels entre chercheurs ou bien par des banques de données conçues à cet effet. C'est ainsi que sont apparues progressivement en linguistique des banques de données accessibles sur Internet : celles-ci restent pour la plupart spécialisées dans l'écrit ou constituées d'oral en grande partie élicité (entretiens ou dispositifs expérimentaux); les banques de données de corpus facilement accessibles à la communauté, constitués de données enregistrées, en audio et en vidéo, d'interactions naturelles ne sont pas nombreuses sur le plan international : on mentionnera d'abord la 
TalkBank ${ }^{4}$, développée par Brian MacWhinney aux USA, qui en est l'exemple le plus ancien, dont les corpus sont unifiés grâce au recours au logiciel CLAN - pour lequel une version spécialement aménagée pour l'AC a été développé avec Johannes Wagner (CLAN_CA). On mentionnera aussi la banque de données CLAPI (Corpus de LAngue Parlée en Interaction) $)^{5}$, développée par le groupe ICOR du laboratoire ICAR à Lyon, qui est une plateforme qui articule une archive de corpus d'interactions en milieu naturel et des moteurs de recherche en permettant l'exploitation outillée.

\section{Vers des analyses systématiques}

Sur la base de ces matériaux recueillis au fil de «naturally occurring interactions », l'AC se caractérise par deux procédures analytiques : la première consiste à proposer une single case analysis, portant sur une donnée qui est parcourue dans sa complexité ; la seconde consiste à effectuer une «analyse de collection » qui se focalise sur un phénomène particulier dans des données différentes et qui vise à en montrer la systématicité. Dans la première, « the resources of past work on a range of phenomena and organizational domains in talk-in-interaction are brought to bear on the analytic explication of a single fragment of talk » (Schegloff, $1987: 101$ ) alors que la seconde consiste à utiliser «a set of fragments, then, to explicate a single phenomenon or a single domain of phenomena » (1987: 101). Alors que la première façon de faire exploite les connaissances analytiques existantes pour comprendre un cas singulier dans sa complexité et pour énoncer des conjectures, la seconde permet d'envisager l'étude d'un objet inédit dans sa systématicité et généralité.

La première procédure a été privilégiée lors de la réception de l'AC : elle renvoie à l'attention pour la dimension indexicale de l'organisation conversationnelle, se traduisant par une attention aux détails particuliers qui fondent l'agencement de tel ou tel extrait enregistré d'un corpus. En revanche, la seconde, qui prolonge la première et s'incarne dans l'élaboration de collections, se traduisant par une exigence de démonstration du caractère récurrent et systématique des phénomènes d'ordre, a été davantage ignorée (cf. Bonu, éd., à paraître pour un panorama sur la production francophone).

Pourtant cet aspect est central dès les premiers travaux de l'AC dans les années 60 , comme en témoignent les Lectures de Sacks (1992). Il y parle de l'objectif de l'AC visant à décrire une «technologie » voire une "machinerie » de la conversation, i.e. un «apparatus », un "ensemble de méthodes » qui puisse renvoyer à la manière dont les instances observées ont été produites. Ces objectifs ont le mérite de clarifier le fait que pour Sacks le but de l'AC n'a jamais été de se limiter à décrire aussi précisément soitil une situation particulière ; le but reste - comme chez Garfinkel - la description de "procédés ", qu'il appelle des «méthodes» (des "ethnométhodes»), qui produisent les instances observées (Schegloff, 1992b : 1338 parle de «procedural infrastructure of interaction »).

Ainsi Sacks caractérise son travail analytique comme visant une description de la façon dont les membres parviennent à produire méthodiquement une action reconnaissable comme telle. Par exemple, en travaillant sur des instances d' « invitations », le focus n'est pas mis sur un «énoncé » en tant que tel qui permettrait de réaliser l'invitation, ou sur l'établissement de définitions générales et constitutives de verbes de parole, à la manière des actes de langage (Schegloff, 1992a, I, xxvi), mais sur les méthodes qui produisent des énoncés reconnaissables et reconnus par les participants comme une invitation (Sacks, II, 367). Ces méthodes permettent éventuellement de distinguer les invitations surgissant de l'interaction («generated in that interaction») des invitations dont l'énoncé est planifié à l'avance, voire qui sont le but de l'interaction (comme les invitations placées dans la raison de l'appel téléphonique) - les deux recevant des réponses différentes et générant ainsi une suite d'actions différente, ayant des trajectoires séquentielles propres (Sacks, 1992, 1, 792-3 ; II, 210).

Cette analyse ne part pas d'un exemple d'invitation typique produit par introspection; elle ne consiste pas à identifier comme point de départ un type d'action. En revanche, elle se penche sur une occurrence particulière, attestée dans un corpus, pour se demander comment elle a été produite méthodiquement $e t$ de manière située (le caractère méthodique étant responsable de la systématicité des procédés, alors que le caractère situé relève de l'agencement indexical et contingent des détails en situation). Une fois identifiée 
et analysée cette occurrence, il s'agit de construire une collection d'occurrences similaires, qui sont reconnues par les participants comme des accomplissements reconnaissables de la même action.

La construction d'une collection vise ainsi à explorer l'organisation récurrente d'un phénomène dans plusieurs fragments de corpus, en obéissant à un certain nombre d'exigences qui fondent la "mentalité analytique » particulière de l'AC et que nous allons rappeler brièvement, en retraçant la manière dont Schegloff (1996a) organise son analyse exemplaire d'une pratique particulière, "confirming allusions ». Il identifie cette pratique en repérant dans son corpus un ensemble de répétitions d'un segment préalable, identifié comme ce qui était visé dans le tour précédent, afin de ratifier les allusions qu'il contenait implicitement/qu'il laissait entendre. Cette répétition est une pratique qui permet de réaliser une action particulière, confirmer les allusions, apparemment minimale et optionnelle mais qui fait partie du « répertoire culturel» des locuteurs (Schegloff 1996a : 209) et qui permet de traiter le thème important de l'implicite. Au moment de commencer l'enquête, Schegloff n'avait pas la moindre idée qu'il pouvait y avoir une action de ce type dans notre société, il ne la présupposait pas, n'en cherchait pas des occurrences pour l'illustrer, ne pouvait pas l'imaginer. D'ailleurs, cette action ne reçoit pas de nom, de catégorie métapragmatique en langue : pour la découvrir, il n'aurait pas pu se baser sur « our vernacular understanding of typicalized couses of action » (Schegloff 1996 : 211). Et pourtant, ce sont de telles actions qui constituent une société dans son fonctionnement moment par moment dans l'interaction (et non telle qu'elle a été définie comme un objet disciplinaire d'étude).

On notera que le "phénomène » ainsi découvert est une «pratique », qui implémente, accomplit, réalise une «action » : en sont des exemples, outre les études de collections d'ouvertures et de confirmation des allusions que nous avons déjà citées, les analyses des évaluations (assessements) par Pomerantz (1984), des réparations par Schegloff (1979, 1992b, etc.), de l'annonce de mauvaises nouvelles par Maynard (1997), de yes/no questions par Koshik (2000), ou de la raison de l'appel téléphonique (Couper-Kuhlen, 2001).

On notera que l'analyse d'un «phénomène » ne porte pas a priori sur une « forme » : plus facilement recherchable dans un grand corpus, une forme ne constitue toutefois pas un point de départ de l' « unmotivated looking ». La raison en est que généralement une forme isolée ne réalise pas en ellemême une action. Les formes du langage sont plutôt des « ressources » qui accomplissent des actions de par leur position particulière au sein d'une pratique et au sein d'une séquence particulière. Même si dans la littérature conversationnelle un certain nombre de formes ont fait l'objet d'analyses systématiques en collections - comme « oh » (Heritage, 1984, 1998, 2002) - il est significatif qu'elles l'aient été en rapport avec un positionnement séquentiel particulier (1984), par exemple en réponse à une question (1998) ou à une évaluation (assessment, 2002) : c'est en tant que réponse à un assessment par exemple que «oh » devient une « méthode » pour moduler l'accord et le désaccord entre les participants ${ }^{6}$.

Une fois identifiée une pratique - qui peut être effectuée en mobilisant des ressources grammaticales récurrentes, mais pas uniquement - il s'agit de vérifier qu'elle est bien une instance de l'action sur laquelle porte la collection, en montrant qu'elle a été reconnue comme telle par les participants. L'orientation des participants - le caractère emic des phénomènes étudiés - fonctionne ainsi comme une procédure de vérification et de preuve, incarnée par et dans les tours suivants, qui manifestent le type d'action accomplie par le tour précédent et sa compréhension par les participants. On voit donc bien que le caractère reconnaissable de l'action pour les participants est étroitement lié aux caractéristiques de l'environnement séquentiel de la pratique étudiée (ce qui précède, ce qui suit, les caractéristiques du formatage du tour, son insertion dans un type de séquence, etc.), qui constitue l'objet de la description systématique.

L'analyse de collections constitue la manière spécifique à l'analyse conversationnelle de produire un ensemble systématique et cumulatif de connaissances sur l'organisation séquentielle des ressources linguistiques en interaction. Ces connaissances sont souvent produites sur la base de grandes masses de données ; si elles donnent lieu à ce que l'on a appelé une quantification "informelle » (expression de fréquences et d'estimations quantitatives sous forme descriptive mais non chiffrée), leur quantification est sujette à d'importantes discussions, qui ne nient pas a priori l'intérêt de ce type de généralisation mais qui 
en interrogent critiquement les conditions. Ainsi dans un article fameux, Schegloff (1993) énonce les problèmes à résoudre préalablement à la quantification - identification d'un dénominateur commun, défini comme l'environnement séquentiel spécifique des occurrences retenues, définition d'un numérateur, ou de la série d'items comptant comme des occurrences du phénomène ; délimitation d'un domaine d'activité organisationnellement pertinent pour le phénomène décrit. Ces préalables montrent que la constitution d'une collection (core collection, une fois résolus les problèmes de boundary instances), au sens technique de l'AC, est bien l'étape analytique indispensable pour pouvoir songer à une quantification (voir Robinson, 2007 sur ce point et Haakana, 2002 pour une discussion sur la possibilité de quantifier les rires) ${ }^{7}$.

\section{Une grammaire-en-interaction encore fragmentaire}

\subsection{Au fondement de l'organisation de l'interaction : la machinerie des tours et les unités de construction du tour}

En décrivant la «machinerie» de l'alternance des tours de parole, Sacks, Schegloff et Jefferson remarquent que a) les locuteurs alternent régulièrement au cours de la conversation, b) généralement une seule partie parle à la fois, c) le passage d'un tour à l'autre se fait en minimisant les chevauchements et les silences (1974: 700). La question ouverte par ces constats apparemment très simples consiste à se demander comment les locuteurs effectuent cette alternance d'un tour à l'autre avec précision, systématicité et méthodicité, sans qu'ils aient à se concerter d'avance ou à en thématiser explicitement le moment et la manière. La réponse à cette question a été cruciale pour l'essor de l'analyse conversationnelle et de la linguistique interactionnelle, puisqu'elle a fondé une analyse des ressources linguistiques telles que traitées par les participants aux fins pratiques de la gestion du turn-taking.

Depuis l'article princeps sur la «machinerie de l'alternance des tours » (Sacks, Schegloff, Jefferson, 1974), la notion de TCU (turn-constructional unit) a été reconnue comme définissant l'unité interactionnelle minimale pour les participants ordonnant leurs contributions à la conversation. Le système du turn-taking se base en effet sur deux composantes, l'une responsable de la composition des tours (turn-constructional component), l'autre de leur allocation (turn-allocation component). La première permet de rendre compte de la façon dont les participants parviennent à identifier le moment où l'alternance du tour peut avoir lieu, en réduisant à la fois les chevauchements et les silences. L'efficacité de la machinerie du turn-taking repose sur une analyse (par les participants) temporellement très précise du déroulement de la parole, permettant la projection de la complétude des unités de construction du tour en train d'émerger dans la parole, dégageant ainsi des possibles points de transition pertinents (transitionrelevance places, TRP) ${ }^{8}$.

La question des TCU est fondamentale pour la linguistique interactionnelle parce qu'elle montre que « given conversation as a major, if not THE major, locus of language's use, other aspects of language structure will be designed for conversational use and, pari passu, for turn-taking contingencies » (Sacks, Schegloff, Jefferson, $1974:$ 722) et que donc non seulement l'organisation interactionnelle exploite la structure des ressources langagières existantes mais aussi, et en retour, que les structures de la langue sont configurées de sorte à remplir leurs fonctionnalités interactionnelles. Dans l'article sur le turn-taking, les TCUs sont définis pour la première fois de cette manière :

There are various unit-types with which a speaker may set out to construct a turn. Unit-types for English include sentential, clausal, phrasal, and lexical constructions (cf. 4.13 below). Instances of the unit-types so usable allow a projection of the unittype under way, and what, roughly, it will take for an instance of that unit-type to be completed. (1974: 702).

As for the unit-types which a speaker employs in starting the construction of a turn's talk, the speaker is initially entitled, in having a turn, to one such unit. The first possible completion of a first such unit constitutes an intial transition-relevance place. 
La première caractérisation des TCUs est donnée en termes syntaxiques par Sacks, Schegloff et Jefferson, dont le modèle « identifies the types of turn-constructional units as sentential, clausal, phrasal, and lexical - i.e. syntactically » : son traitement « should indicate the deep ways in which syntax matters to turntaking, albeit a syntax conceived in terms of its relevance to turn-taking. » (1974: 720-721). Cette primauté de la syntaxe est complétée par la référence à la prosodie : «Clearly, in some understanding of 'sound production' (i.e. phonology, intonation, etc.), it is also very important to turn-taking organization. For example, discriminations between "what" as a one-word question and as the start of a sentential (or clausal or phrasal) construction are made not syntactically but intonationally. When it is further realized that any word can be made into a 'one-word' unit-type, via intonation, then we can appreciate the partial character of the unit-types' description in syntactic terms » (1974: 721-722).

\subsection{Conséquences pour la description de la grammaire en interaction}

Cette caractérisation initiale des TCUs a porté au développement d'une littérature importante, qui a développé plusieurs paliers d'analyse, en insistant sur les interfaces entre eux :

- une syntaxe pour l'interaction, fondée sur une conception 'incrémentale" et projective de la syntaxe (Auer, 2005) ${ }^{10}$ : elle rend compte de la manière dont les constructions syntaxiques se construisent en temps réel, pas à pas, au fil du déroulement des activités interactionnelles des participants et en étant sensible à leurs contingences. Au sein de cette syntaxe, les constructions sont décrites par rapport à leur potentiel projectif (Schegloff, 1996 ; Auer, 2002) ${ }^{11}$.

- des réflexions sur l'articulation entre syntaxe et prosodie (Ford \& Thompson, 1996, Selting, 2000), avec des discussions sur la priorité éventuelle de l'une sur l'autre - donnant lieu au développement important de la prosodie interactionnelle (Couper-Kuhlen \& Selting (eds) 1996) ainsi que de la phonétique interactionnelle (Local \& Kelly, 1986, Local et alii, 1986).

- des réflexions sur le fait que le TCU est localement défini par un cluster de dimensions à différents niveaux (Ford, Fox, Thompson, 1996), que les participants traitent de manière holiste : outre les dimensions syntaxique et prosodique, interviennent les dimensions sémantique (la complétude envisagée du point de vue du sens) et pragmatique (la complétude entendue du point de vue de l'action réalisée par l'unité, cf. Ford \& Thompson, $1996: 148)$, auquel s'ajoute plus tardivement la multimodalité (mais voir déjà Ford, Fox, Thompson, 1996).

- des réflexions sur la contribution de la multimodalité à cette structuration linguistique des TCU et des tours (Goodwin, 1981 ; Streeck \& Hartge, 1992, Mondada, 2004, 2007a ; Schmitt, 2005) constituant un terrain fertile pour l'étude des projections et des anticipations qui structurent de manière flexible les tours comme les TCUs et définissent dynamiquement la complétude d'unités à plusieurs niveaux.

Du point de vue de la linguistique interactionnelle, il ne faut pas oublier que la définition des TCUs et des TRPs reste un accomplissement pratique des membres - et que cette définition praxéologique prime sur les considérations formelles qu'il serait possible d'énoncer sur la complétude de l'unité. Cela signifie que les propriétés formelles du tour ne sont pas un ensemble de paramètres pouvant être évalués de manière exogène par l'analyste, mais plutôt un ensemble de ressources des participants, soumises à leur appréciation. Autrement dit, les TCUs concernent moins des structures formelles ${ }^{12}$ que des pratiques méthodiquement organisées des locuteurs (Ford, Fox, Thompson, $1996: 431$; Ford, 2004) ${ }^{13}$.

Cette dimension praxéologique a comme conséquence que plutôt que de vouloir circonscrire des unités, l'objectif de l'analyse est de caractériser des pratiques de construction du tour et de l'activité effectuée dans et par le tour; plutôt que de viser des critères nécessaires et suffisants de segmentation d'unités, d'identifier des pratiques qui accomplissent la complétion ou la continuation d'une unité.

De ce point de vue, des convergences aussi bien que des divergences entre dimensions sont observables : alors que la syntaxe peut indiquer une articulation en plusieurs unités, la prosodie, la relation séquentielle avec ce qui précède ou la gestualité peuvent indiquer une continuation de l'unité : ces divergences 
peuvent être amplifiées par l'interprétation en temps réel des co-participants, qui peuvent privilégier l'une ou l'autre lecture aux fins pratiques de leur propre action - par exemple pour prendre ou ne pas prendre le tour.

En particulier, le locuteur peut intervenir sur la forme qu'est en train de prendre le tour et ses TCUs, en la transformant - par exemple en produisant une expansion ou bien en choisissant un format plus compact (Sacks, Schegloff, Jefferson, 1974: 726), qui changent les conditions auxquelles les co-participants peuvent ou non prendre la parole.

Ainsi, Local \& Kelly (1986) montrent que le silence suivant une conjonction peut être configuré de sorte à faire l'objet de deux analyses possibles mais opposées: d'une part les participants peuvent traiter ce silence comme projetant quelque chose à venir de la part du locuteur (et, dans ce cas, comme ne représentant pas des TRP); d'autre part, ce silence peut être produit, grâce à des caractéristiques phonétiques spécifiques, comme permettant une alternance du tour. Dans un cas on pourra le traiter comme une pause appartenant au tour in fieri, dans l'autre comme une pause inter-tour offrant un TRP.

Cela produit, en définitive, une «ultimate 'indefinability' of TCUs [which] is essential to their functionality. Interactants regularly extend, foreshorten, reanalyze, and repair their developing turns in response to contingencies emergent at particular points in particular conversations » (Ford, Fox, Thompson, $1996: 428)$; «thus projection creates a manipulable potential end point, a provisional and negotiable goal that can be confirmed or manipulated through the same practices that produced it in the first place » $(1996: 449)$.

\subsection{Développements ultérieurs}

Plus généralement, l'essor de la linguistique interactionnelle permet aujourd'hui d'interroger de manière cohérente la relation entre

- des ressources linguistiques (des configurations formelles, des formes lexicales, des constructions syntaxiques) et multimodales (dans des associations entre par exemple certaines formes verbales et des phénomènes gestuels ou visuels con-comitants),

- des environnements séquentiels (des positions au sein du tour, de la séquence ou de l'activité),

- des actions accomplies par les participants.

Si la démarche consistant à constituer des collections invite à articuler étroitement ces trois dimensions, on remarque au sein de la linguistique interactionnelle contemporaine des variations - relevant souvent de la manière dont les auteurs se positionnent vis-à-vis d'autres recherches en linguistique et définissent par conséquent leur objet - quant à la dimension qui est privilégiée et qui structure l'analyse.

Une première stratégie consiste à se focaliser sur une forme, ensuite explorée dans les séquences qui la caractérisent et les actions qu'elle contribue à effectuer : cela représente une manière de procéder alignée avec la tradition linguistique, permettant dans certains cas de revisiter le traitement fonctionnel qui en a été proposé par elle (cela a été privilégié par les travaux sur les connecteurs et marqueurs linguistiques, comme « actually », Clift, 2001 ; « obwohl » Günther, 1999; en français « ben » ou « voilà » Bruxelles et Traverso, 2001, 2006, Groupe ICOR, 2008 ; «attends », Groupe ICOR 2007, ou par les travaux sur certaines constructions syntaxiques, comme en français les dislocations à gauche, Fornel, 1988, Pekarek, 2001 ou la dislocation à droite, Horlacher, 2008).

Une seconde stratégie consiste à se focaliser sur des séquences particulières : cette manière de procéder s'aligne avec la tradition de l'analyse conversationnelle et privilégie des environnements séquentiels particuliers, que ce soient des positionnements séquentiels au sein du tour (Schegloff, 1996b, Auer, 1996 ; en français Mondada, 2007b, à paraitre), des séquences spécifiques (voir par exemple les nombreuses analyses des séquences de réparation, et leur contribution à l'étude de la syntaxe : Schegloff, 1979, Fox \& Jasperson, 1995, Egbert, 1997, Wu, 2007; en français voir les travaux de Gülich, 1986, Gülich \& 
Kotschi, 1987 sur la reformulation), ou des phénomènes séquentiels particuliers (comme les chevauchements, Schegloff, 2000, en français Oloff, à paraître).

Une troisième angle d'approche consiste à adopter comme point de départ une action ou la structuration d'une activité, pour y analyser les types de ressources mobilisées pour les accomplir (par exemple les plaintes, Drew, 1998, les offres Curl, 2006 ; par exemple la structuration des activités en transitions d'un épisode à un autre, Bruxelles, Greco, Mondada, à paraître, Mondada, 2006c, Mondada \& Traverso, 2005, en séquences d'ouverture, Schegloff, 1968 ; en français et en allemand Mondada \& Schmitt à paraître ; ou de clôture, Schegloff \& Sacks, 1973, en italien De Stefani, 2006). L'analyse multimodale complexifie encore davantage ce paysage : les analyses des ressources gestuelles, visuelles, actionnelles se focalisent sur des clusters de phénomènes concomitants et coordonnés au sein d'actions particulières, sans oublier la matérialité et la spatialité du contexte où elles sont accomplies (sur l'importance des déplacements, par exemple, voir une réanalyse de la deixis spatiale Mondada, 2005b, du rapport entre construction du tour et déplacement dans l'espace, Relieu, 1999, du rapport entre mouvement et organisation des tours De Stefani \& Mondada, 2007).

Ces différentes options ne se contredisent pas en principe mais produisent des développements cumulatifs distincts, ayant des implications théoriques différentes pour la pensée et l'approche de la langue en interaction.

\title{
6 Conclusion : une approche émergentiste du langage
}

Une telle conception de la parole en interaction relève plus généralement d'une approche des structures du langage qui considère non seulement que les premières sont sélectionnées, mobilisées, exploitées de manière fonctionnelle à l'interaction mais, plus radicalement, qu'elles sont configurées, en tant que structures, d'une manière sensible à leur usage prototypique dans l'interaction. Comme le dit Schegloff,

\begin{abstract}
« it would hardly surprise us if some of the most fundamental features of natural language are shaped in accordance with their home environment in copresent interaction, as adaptations to it, or as part of its very warp and weft (Schegloff, 1989 : $142-4,1991: 153-5)$. For example, if the basic natural environment for sentences is in turns-at-talk in conversation, we should take seriously the possibility that aspects of their structure - for example, their grammatical structure - are to be understood as adaptations to that environment. In view of the thoroughly local and interactional character of the organization of turn-taking in conversation (SSJ, 1974), the grammatical structures of language should in the first instance be understood as at least partially shaped by interactional considerations (Schegloff, 1979). And one locus of those considerations will be the organization of the turn, the organizational unit which 'houses' grammatical units » (1996a : 54-55).
\end{abstract}

Cette vision est proche de celle défendue par Hopper de la « grammaire émergente » (1987), par laquelle il critique une grammaire basée sur un système fixe voire figé, pour inviter à penser la grammaire en relation avec les expériences des locuteurs en action, comme répondant aux contraintes et aux pressions exercées par le discours. Dans la perspective interactionnelle, cela amène à considérer que "it seems appropriate that grammar would arise from, or emerge from, a dynamic constellation of interactional practices » $(\text { Fox, } 2007: 302)^{14}$. Cette conception émergentiste peut se penser à deux niveaux distincts mais interreliés :

- au niveau de l'organisation des énoncés et des tours de parole en interaction, pensables en tant que se structurant progressivement au fur et à mesure que progresse la production du tour, de manière incrémentale, enrichie mais aussi negociée, confirmée, ou déviée par les contingences de la temporalité de l'interaction. Ce niveau permet d'appréhender les choix linguistiques situés des locuteurs dans leur action - et donc leurs innovations langagières individuelles, plus ou moins conjoncturelles. Dans cette émergence événementielle, on peut décrire l'apparition de nouvelles constructions syntaxiques, qu'elles soient ou non retenues par le cours de l'histoire (soit parce que les locuteurs cessent de parler ainsi, soit parce que les institutions rejettent ces 
constructions nouvelles). Ce qui importe pour l'émergence conjoncturelle, ce n'est pas la longévité des formes mais la manière dont elles viennent au jour.

- De plus, la notion de grammaire émergente est utile au niveau de comportements systématiques et répétitifs de Gestalten constituées par des formes particulières observables dans des environnements séquentiels et actionnels particuliers. Ces Gestalten constituent des ensembles de formes-dans-des-actions qui dépassent les innovations individuelles. Leur récurrence permet d'observer une stabilisation dans certains contextes - décrits en respectant les ajustements à des contraintes praxéologiques particulières. Ils peuvent constituer le début d'un processus de grammaticalisation (cf. « it seems appropriate that grammar would arise from, or emerge from, a dynamic constellation of interactional practices » Fox, 2007 : 302) (cf. Günthner, 1999, Gohl \& Günthner, 1999 ; Groupe ICOR, 2007 pour des exemples).

Une première conséquence en est que la grammaire est conçue comme un ensemble de ressources interactionnelles, non seulement actualisées, non seulement mobilisées mais aussi configurées en réponse aux contingences de l'interaction; cette grammaire partage avec les unités interactionnelles les caractéristiques de l'indexicalité, de la plasticité, de l'ajustement aux contraintes praxéologiques.

Une seconde conséquence concerne la définition même de ces ressources : s'il s'agit de considérer les ressources effectivement mises en oeuvre par les locuteurs dans l'organisation de leurs tours en interaction, alors il n'y a aucune raison de séparer d'un côté ressources «verbales » et de l'autre ressources «non-verbales $»^{15}$. Une approche de la multimodalité permet précisément de traiter ensemble les ressources grammaticales, prosodiques et corporelles (gestes, regards, mimiques faciales, mouvements de la tête, postures corporelles, etc.) que les participants mettent en oeuvre de manière holiste dans l'organisation méthodique de leur conduite, dans une orientation commune vers les opportunités et les contraintes émergeant de l'interaction. Une telle conception des ressources ne se situe pas en dehors de la linguistique mais la rend simplement moins logocentrique.

\section{Références}

Auer, P. (1996). The pre-front field in spoken German and its relevance as a grammaticalization position. Pragmatics, 6(3), 295-322.

Auer, P. (2002), Projection in interaction and projection in grammar. InLiSt - Interaction and Linguistic Structure (Universität Potsdam), no 33, 1-43 (http://www.uni-potsdam.de/u/inlist/).

Auer, P. (2005). Syntax als Prozess. InLiSt - Interaction and Linguistic Structure (Universität Potsdam), no 41, 1-35 (http://www.uni-potsdam.de/u/inlist/).

Bonu, B. (1992). L'analyse de conversation: une discipline de l'action sociale. Quaderni, 17, 51-64.

Bonu, B. (éd.) (2002). Numéro spécial sur la transcription de l'interaction. Cahiers de Praxématique, 39.

Bonu, B. (éd.) (à paraître). Numéro spécial sur la comparaison en analyse conversationnelle. Cahiers de Praxématique.

Brown, P. \& S.C. Levinson (1978) Universals in language usage: Politeness phenomena. In Esther N. Goody (ed.) Questions and Politeness, pp. 56-289. Cambridge: Cambridge University Press.

Bruxelles S. \& Traverso V. (2002). Ben dans deux situations polylogales. Apport de la description d'un "petit mot" du discours à l'étude des polylogues. Marges Linguistiques 2.

Bruxelles, S., \& Traverso, V. 2006). Usages de la particule voilà dans une réunion de travail : analyse multimodale. In : M. Drescher \& B. Job (eds). Les marqueurs discursifs dans les langues romanes : approches théoriques et méthodologiques. Bern : Peter Lang, 71-92.

Bruxelles S., Greco L., Mondada L. (à paraître). Pratiques de transition : ressources multimodales pour la structuration de l'activité. In: F. Détienne, \& V. Traverso (éds.), Méthodologie d'analyse de situations coopératives de conception, Nancy : PUN. 
Bürki, Y. \& E. De Stefani (éds.). Trascrivere la lingua. Dalla filologia all'analisi conversazionale / Transcribir la lengua. De la Filología al Análisis Conversacional. Bern, Peter Lang.

Clayman, S. et alii (2006). Historical Trends in Questioning Presidents 1953-2000. Presidential Studies Quarterly 36: 561-583.

Clift, R. (2001). Meaning in interaction: the case of actually. Language 77 (2): 245-291.

Couper-Kuhlen, B., Selting, M. (eds.). (2001). Studies in Interactional Linguistics. Amsterdam: Benjamins.

Couper-Kuhlen, E. (2001). Constructing reason-for-the-call turns in everyday telephone conversation, InLiSt Interaction and Linguistic Structures, 25, (http://www.uni-potsdam.de/u/inlist/issues/25/index.htm)

Couper-Kuhlen, E., Ford, C.E. (eds.) (2004), Sound Patterns in Interaction. Amsterdam: Benjamins.

Couper-Kuhlen, E., Selting, M. (eds.) (1996). Prosody in Conversation: Interactional studies. Cambridge: Cambridge University Press.

Curl, T. (2006). Offers of assistance: Constraints on syntactic design. Journal of Pragmatics, 38(8) : 1257-1280.

De Stefani, E. \& L. Mondada (2007). Muoversi nello spazio. Le interazioni di coppia in macchina e al supermercato. In : E. De Stefani (éd.), Regarder la langue. Les données vidéo dans la recherche linguistique. Bulletin Suisse de Linguistique Appliquée 85 : 131-159.

De Stefani, E. (2006a). Le chiusure conversazionali nell'interazione al banco di un supermercato. In : Y. Bürki \& E. De Stefani (éds.). Trascrivere la lingua. Dalla filologia all'analisi conversazionale / Transcribir la lengua. De la Filología al Análisis Conversacional. Bern : Peter Lang, 369-403.

Drew, P., \& Heritage, J. (Eds.). (1992). Talk at Work. Cambridge: Cambridge University Press.

Drew, P. (1998). Complaints About Transgressions and Misconduct. Research on Language and Social Interaction, 31(3-4) : 295-325.

Egbert, M. M. (1997). Some interactional achievements of other-initiated repair in multiperson conversation. Journal of Pragmatics, $27: 611-634$.

Ford, C. E. (2004). Contingency and units in interaction. Discourse Studies, 6(1) : 27-52

Ford, C.E., Fox, B., \& Thompson, S.A. (1996). Practices in the construction of turns : the «TCU » revisited. Pragmatics, $6 / 3: 427-454$.

Ford, C.E., Fox, B.A. \& Thompson, S.A. (eds) (2002). The Language of Turn and Sequence. Cambridge: Cambridge University Press.

Ford, C.E., \& Thompson, S.A. (1996). Interactional units in conversation : Syntactic, intonational, and pragmatic resources for the management of turns. In E. Ochs, E.A. Schegloff, S.A. Thompson (eds.). Interaction and Grammar. Cambridge : Cambridge University Press, 134-184.

Fornel, M., de \& J. Léon (2000). L'analyse de conversation. De l'ethnométhodologie à la linguistique interactionnelle. Histoire Épistémologie Langage 22/1 : 131- 155.

Fornel, M. de (1988). Constructions disloquées, mouvement thématique et organisation préférentielle dans la conversation. Langue Française 78 : 101-123.

Fox, B. (2007). Principles shaping grammatical practices : an exploration. Discourse Studies, 9 : 299-318.

Fox, B., \& Jasperson, R. (1995). A syntactic exploration of repair in conversation. In P. Davis (Ed.), Descriptive and Theoretical Modes in the Alternative Linguistics (pp. 77-134). Amsterdam: Benjamins.

Garfinkel, H. (1967). Studies in Ethnomethodology. Englewood Cliffs, N.J.: Prentice-Hall.

Gohl, C. \& Günthner, S. (1999). Grammatikalisierung von ,weil’ als Diskursmarker in der gesprochenen Sprache. Zeitschrift für Sprachwissenschaft 18 (1) : 39-75.

Goodwin, C. (1979). The Interactive Construction of a Sentence in Natural Conversation. In : Psathas, G. (ed.). Everyday Language: Studies in Ethnomethodology (pp. 97-121). New York: Irvington Publishers.

Goodwin, C. (1981). Conversational Organization: Interaction Between Speakers and Hearers. New York: Academic Press. 
Goodwin, C. (2007). Interactive footing. In : E. Holt \& R. Clift (eds.). Reporting Talk: Reported Speech in Interaction (pp. 16-46). Cambridge: Cambridge University Press.

Goodwin, C., Goodwin, M.H. (2004). Participation. In Duranti, A. (ed.). A Companion to Linguistic Anthropology. Oxford: Basil Blackwell.

Goodwin, M.H. (1990). He-Said-She-Said: Talk as Social Organization among Black Children. Bloomington: Indiana University Press.

Groupe ICOR (L. Balthasar, S. Bruxelles, L. Mondada et V. Traverso) (2007). Variations interactionnelles et changement catégoriel : l'exemple de 'attends'. In : Auzanneau M. (éd.), La mise en oeuvre des langues dans l'interaction. Paris : L'Harmattan, 299-319.

Groupe ICOR (M. Bert, S. Bruxelles, C. Etienne, L. Mondada, V. Traverso) (2008). Browsing and searching interactional corpora on the CLAPI database: the case of 'voilà'. Journal of French Language Studies, 18/1 : 121145.

Gülich E. (1986). 'Soûl ce n'est pas un mot très français'. Procédés d'évaluation et de commentaire méta-discursif dans un corpus de conversations en situations de contact. Cahiers de linguistique française, $7: 231-258$.

Gülich, E. (1991). Pour une ethnométhodologie linguistique. Description des séquences conversationnelles explicatives. In : Dausendschön-Gay, U.; Gülich, E.; Krafft, U. (eds.). Linguistische Interaktionsanalysen. Tübingen : Niemeyer Verlag.

Gülich, E. \& Kotschi, T. (1987). Les actes de reformulation dans la consultation: La dame de Caluire. In : P. Bange (éd.). L'analyse des interactions verbales. Lyon: PUL.

Gülich, E., Mondada, L. (2001). Analyse conversationnelle. In : G. Holtus, M. Metzeltin, C. Schmitt (eds). Lexikon der romanistischen Linguistik, Tübingen: Niemeyer, Band I,2, 196-250.

Gumperz, J. (1982). Discourse Strategies. Cambridge: C.U.P.

Günthner, S. (1999). Entwickelt sich der Konzessivkonnektor 'obwohl' zum Diskursmarker ? Grammatikalisierungstendenzen im gesprochenen Deutsch. Linguistische Berichte, 180, 409-446.

Haakana, M. (2002). Laughter in medical interaction: from quantification to analysis, and back. Journal of Sociolinguistics, 6(2) : 207-235.

Hakulinen, A., Selting, M. (eds.) (2005). Syntax \& Lexis in Conversation. Amsterdam: Benjamins.

Harris, R. (1981). The Language Myth. London: Duckworth.

Hayashi, M. (1997). An exploration of sentence-final uses of the quotative particle in Japanese spoken discourse. Japanese/Korean Linguistics Vol. 6. (pp. 565-581). Standord: CSLI Publicatons.

Heritage, J. (1984). A change-of-state token and aspects of its sequential placement. In : J. M. Atkinson \& J. Heritage (eds.). Structures of Social Action. Studies in Conversation Analysis (pp. 299-345). Cambridge: Cambridge University Press.

Heritage, J. (1998). Oh-prefaced Responses to Inquiry. Language in Society, 27(3): 291-334.

Heritage, J. (2002). Oh-prefaced responses to assessments: a method of modifying agreement/disagreement. In: C. Ford, B. Fox \& S. Thompson (eds). The Language of Turn and Sequence, New York, Oxford University Press.

Holt, E. (1997). Reporting on Talk: The Use of Direct Reported Speech in Conversation. Research on Language and Social Interaction 29(3): 219-245.

Holt, E. \& R. Clift (eds.). Reporting Talk: Reported Speech in Interaction, Cambridge: Cambridge University Press.

Hopper, P. (1987). Emergent grammar. Berkeley Linguistics Conference (BLS), 13, 139-157.

Horlacher, S. (à paraître). La dislocation à droite. Numéro spécial sur la Structuration grammaticale et la structuration discursive, Revue Tranel, 47.

Kerbrat-Orecchioni, C. (1990-1994). Les Interactions verbales. Paris : Colin, 3 vols.

Koshik, I. 2002. A conversation analytic study of yes/no questions which convey reversed polarity assertions. Journal of Pragmatics 34:1851-1877. 
Lerner, G. (Ed.). (2004). Conversation Analysis. Papers from the First Generation. Amsterdam: Benjamins.

Linell, P. (2005). The Written Language Bias in Linguistics: Its Nature, Origins and Transformations. London \& New York: Routledge.

Local, J. K., Kelly, J., \& Wells, W. H. G. (1986). Towards a phonology of conversation: turn-taking in urban Tyneside speech. Journal of Linguistics, 22(2), 411-437.

Local, J., Kelly, J. (1986). Projection and 'silences' : notes on phonetic and conversational structure. Human Studies, 9, 185-204.

Maynard, D.W. (1997). The news delivery sequence: Bad news and good news in conversational interaction. Research on Language and Social Interaction, 30, 93-130.

McQuown, N. A. (ed). (1971): The Natural History of an Interview. Chicago, University of Chicago Library, Microfilm collection of manuscripts on cultural anthropology, no 95, serie xv.

Mondada, L. (1998/2001). Pour une linguistique interactionnelle. Acta Romanica Basiliensa ARBA, 8, 1998, 113130. Version élargie publiée dans Marges Linguistiques, 1, 2001.

Mondada, L. (2000). Les effets théoriques des pratiques de transcription. LINX, $42: 131-150$.

Mondada, L. (2004). Temporalité, séquentialité et multimodalité au fondement de l'organisation de l'interaction : Le pointage comme pratique de prise du tour. Cahiers de Linguistique Française, 26 : 169-192.

Mondada, L. (2005a). L'analyse de corpus en linguistique interactionnelle : de l'étude de cas singuliers à l'étude de collections, In. A. Condamine (éd.), Sémantique et corpus (pp. 76-108). Paris : Hermès.

Mondada, L. (2005b). La constitution de l'origo déictique comme travail interactionnel des participants: une approche praxéologique de la spatialité. Intellectica. No spécial sur Espace, Inter-action \& Cognition, 41-42/2-3 : $75-100$.

Mondada, L. (2006a). La pertinenza del dettaglio: registrazione e trascrizione di dati video per la linguistica interazionale. In : Y. Bürki, E. de Stefani (eds). Trascrivere la lingua. Dalla filologia all'analisi conversazionale. Bern : Lang, 313-344.

Mondada, L. (2006b). Video Recording as the Preservation of Fundamental Features for Analysis. In : Knoblauch, H., Raab, J., Soeffner, H.-G., Schnettler, B. (eds). Video Analysis. (51-68). Bern : Lang.

Mondada, L. (2006c). Participants' online analysis and multimodal practices: projecting the end of the turn and the closing of the sequence. Discourse Studies $8: 117-129$.

Mondada, L. (2007a). Transcript variations and the indexicality of transcribing practices. Discourse Studies 9/6 : 809-821.

Mondada, L. (2007b). Multimodal resources for turn-taking: Pointing and the emergence of possible next speakers. Discourse Studies, 9/2 : 195-226.

Mondada, L. (à paraître). Ressources linguistiques, vocales et gestuelles pour l'organisation des pre-beginnings : analyse systématique et conséquences pour la constitution de corpus interactionnels. In: Actes du Colloque Corpora Romanica, Freiburg, sept. 2006, Tübingen : Niemeyer.

Mondada, L. \& Schmitt, R. (à paraître). Openings revisited.

Mondada, L., \& Traverso, V. (2005). (Dés)alignements en clôture : une étude interactionnelle de corpus de français parlé en interaction. Lidil, no spécial Corpus oraux et diversité des approches, $31: 34-59$.

Ochs, E., Schegloff, E.A., Thompson, S.A. (eds.) (1996). Interaction and Grammar. Cambridge : Cambridge University Press.

Oloff, F. (à paraître). Établir une collection pour l'étude des chevauchements. Comparabilité de données audio et vidéo. Cahiers de praxématique.

Pekarek Doehler, S. (2001). Dislocation à gauche et organisation interactionnelle. Marges Linguistiques, 2, 177-194. http://www.marges-linguistiques.com/

Pekarek Doehler, S. (2005). Grammaire, discours, interaction: vers une approche interactionniste des ressources grammaticales liées à l'organisation discursive. TRANEL (Travaux Neuchâtelois de Linguistique), 41, 1-15. 
Pomerantz, A. (1984). Agreeing and disagreeing with assessments: some features of preferred/dispreferred turn shapes. In: Atkinson, J. M., J. Heritage (eds.). Structures of Social Action: Studies in Conversation Analysis. (pp. 57-101). Cambridge: Cambridge University Press.

Psathas, G. (1995). Conversation Analysis: The Study of Talk-in-Interaction. Thousand Oaks: Sage.

Relieu, M. (1999). Parler en marchant. Pour une écologie dynamique des échanges de paroles. Langage et Société, $89: 37-69$.

Relieu, M., \& Brock, F. (1995). L'infrastructure conversationnelle de la parole publique, Politix 35 : 77-112.

Robinson, J. (2007). The Role of Numbers and Statistics within Conversation Analysis, Communication Methods and Measures $1 / 1: 65-75$.

Roulet, E. (1980). Stratégies d'interaction, modes d'implicitation et marqueurs illocutoires. Cahiers de linguistique française. 1, 80-103.

Roulet, E. et alii (1985). L'articulation du discours en Français contemporain. Bern, Lang.

Sacks, H. (1984). Notes on methodology. In : J.M. Atkinson \& J. Heritage (eds.). Structures of Social Action. (pp. 21-27). Cambridge, CUP.

Sacks, H. (1992). Lectures on conversation. Oxford : Blackwell, 2 voll.

Sacks, H., Schegloff, E.A. (2002 [1971]. Home position. Gesture 2 : 133-146.

Sacks, H., Schegloff, E.A. \& Jefferson, G. (1974). A Simplest Systematics for the Organization of Turn Taking for Conversation, Language 50(4): 696-735.

Schegloff, E.A. (1968). Sequencing in Conversational Openings. American Anthropologist 70 : 1075-1095.

Schegloff, E.A. (1979). The relevance of repair for syntax for conversation. In: T. Givon (ed.). Syntax and Semantics. Vol. 12: Discourse and Syntax. (pp. 261-288). New York: Academic Press.

Schegloff, E.A. (1982). Discourse as an interactional achievement : some uses of « uh huh » and other things that come between sentences. In : D. Tannen (ed.). Analyzing Discourse : Text and Talk. (pp. 71-93). Georgetown : Georgetown University Press.

Schegloff, E.A. (1987). Analyzing single episode of conversation : an exercice in conversation analysis. Social Psychology Quarterly 50 : 101-114.

Schegloff, E.A. (1992a). Introduction to H. Sacks, Lectures on Conversation. Oxford: Basil Blackwell.

Schegloff, E.A. (1992b). Repair after next turn: The last structurally provided defense of intersubjectivity in conversation. American Journal of Sociology, 98: 1295-1345.

Schegloff, E. (1993). Reflections on Quantification in the Study of Conversation. Research on Language and Social Interaction $26 / 1: 99-128$.

Schegloff, E. A. (1996a). Confirming allusions. American J. of Sociology 102(1), :161-216.

Schegloff, E. A. (1996b). Turn organization: One intersection of grammar and interaction. In: E. Ochs, E. A. Schegloff \& S. A. Thompson (eds.). Grammar and Interaction (pp. 52-133). Cambridge: Cambridge University Press.

Schegloff, E.A. (2000). Overlapping talk and the organization of turn-taking for conversation. Language in Society, 29 (1).

Schegloff, E.A. (2007). Sequence Organization in Interaction: A Primer in Conversation Analysis. Cambridge: Cambridge University Press.

Schegloff, E. A., Jefferson, G., \& Sacks, H. (1977). The preference for self-correction in the organization of repair in conversation. Language, $53: 361-382$.

Schegloff, E.A. \& Sacks, H. (1973). Opening Up Closings, Semiotica 8/4 : 289-327.

Schmitt, R. (2005). Zur multimodalen Struktur von turn-taking. Gesprächsforschung - Online-Zeitschrift zur verbalen Interaktion. $6: 17-61$ (www.gespraechsforschung-ozs.de). 
Selting, M. (2000). The Construction of Units in Conversational Talk. Language in Society 29: 477-517.

Selting, M., Couper-Kuhlen, B. (2001a). Argumente für die Entwicklung einer interaktionalen Linguistik. Gesprächsforschung 1: 76-95.

Selting, M., Couper-Kuhlen, B. (2001b). Forschungsprogramm,Interaktionale Linguistik'. Linguistische Berichte, $187,257-287$.

Sinclair, J. M. \& R.M. Coulthard (1975). Towards an analysis of discourse. Oxford Univ. Press.

Streeck, J., \& Hartge, U. (1992). Previews: Gestures at the transition place. In P. Auer \& A. di Luzio (eds.). The Contextualization of Language. Amsterdam: Benjamins.

Volosinov, V.N. (1929/1973). Marksizm i filosofija jazyka, Moscow: Labirint, 1929; trad. angl. Marxism and the Philosophy of Language. New York: Seminar, 1973.

$\mathrm{Wu}, \mathrm{R}$. (2006). Initiating repair and beyond : the use of two repeat-formatted repair initiations in Mandarin conversation. Discourse Prosesses 41(1) : 67-109.

\footnotetext{
${ }^{1}$ Les lectures de Bakhtine et de Volosinov ont suscité des débats entre ceux qui privilégient le dialogisme propre à la voix polyphonique des auteurs et ceux qui privilégient l'interactivité propre à l'échange entre les co-participants : les travaux de C. Goodwin (2007), M.H. Goodwin (1990), Goodwin \& Goodwin (2004) ainsi que de Holt (1997), Hayashi (1997) et la collection de textes publiés par Holt \& Clift (2007) montrent l'intérêt d'aborder les différentes formes de polyphonie et le discours rapporté dans l'interaction et de dépasser ce clivage.
}

2 On relèvera que dix ans plus tôt, Gülich (1991) parlait, de manière plus radicale, d' « ethnométhodologie linguistique $» .$.

${ }^{3}$ Pour des introductions voir Bonu (1992), Relieu \& Brock (1995), Fornel \& Léon (2000), Gülich \& Mondada (2001).

${ }^{4}$ http://talkbank.org. Le site présente aussi des indications sur les bonnes pratiques et sur la technologie des corpus.

${ }^{5}$ http://clapi.univ-lyon2.fr. Cette banque de donnée est associée à un site, CORINTE (corpus d'interactions) qui expose les principes de constitution des corpus, depuis leur recueil, jusqu'à leur analyse en passant par leur transcription et préparation pour l'exploitation outillée : Voir http://icar.univ-lyon2.fr/projets/corinte/.

${ }^{6}$ Pour une autre analyse, exemplaire, d'une forme et des actions qu'elle implémente voir Clift (2001) sur « actually ».

${ }^{7}$ La question de la quantification a été explorée de différentes manières : Ford, Fox, Thompson (1996) quantifient les propriétés syntaxiques, prosodiques et pragmatiques des TCUs pour identifier des convergences ou des complémentarité dans les ressources qui accomplissenet la reconnaissabilité de la complétude des unités ; Clayman et alii (2006) codent des formats de question pour une analyse longitudinale et historique de l'évolution des pratiques de questionnement des journalistes face aux présidents américains. Si ces décomptes sont effectués manuellement, l'accessibilité de grands corpus dans des banques de données dotées d'outils de navigation performants permet d'envisager des formes semi-automatiques d'explorations de corpus : ainsi le groupe $\operatorname{ICOR}(2007,2008)$ utilise de manière heuristique des identifications automatiques de clusters de phénomènes (formes linguistiques + caractéristiques temporelles de l'interaction + positions séquentielles) pour identifier et vérifier des occurrences candidates à constituer des collections.

${ }^{8}$ Les deux notions de TCU et de TRP sont ainsi liées : "The end of any such [turn-constructional] unit is a possible completion of a turn, and possible completion of turns are places at which potential next speakers appropriately start next turns » (Schegloff, $1982: 75$ ).

9 «Inkrementelle Syntax beschreibt die fortlaufenden Projektionen über den weiteren Verlauf der emergenten syntaktischen Struktur, die es den Hörern erlauben, den entstehenden Redebeitrag ohne Verzögerung zu prozessieren. Syntaktische Projektionen bauen auf syntaktischen 'Gestalten' auf, die, sobald sie identifiziert sind, nach dem gestaltpsychologischen Prinzip der ,guten Fortsetzung“ durch die Produktion einer mehr oder weniger präzise vorhersagbaren Abschlussstruktur geschlossen werden müssen. Syntaktische Projektionen sind für die Interaktion nicht zuletzt deshalb von großer Wichtigkeit, weil sie die Vorhersage von möglichen Redezug- Abschlusspunkten ermöglichen. Sie kommen aber auch innerhalb von Turnkonstruktionseinheit zum Tragen » (Auer, $2005: 3$ ).

${ }^{10}$ Ce type d'analyse a évidemment contribué à la critique de la syntaxe basée sur la notion de phrase et au développement d'une syntaxe basée sur les pratiques des locuteurs (cf. Ford \& Thompson, 1996 : 143-44). 
11 Ce type de syntaxe entretient des liens traditionnels avec la syntaxe fonctionnelle (voir l'introduction à Ochs, Schegloff, Thompson, 1996) et avec la " construction grammar », bien que des différences notables séparent les deux modèles (voir Depperman, 2006).

12 Par rapport à la syntaxe, Ford, Fox \& Thompson (1996) remarquent que cela a deux conséquences importantes : d'une part il s'agit de tenir compte d'une syntaxe située, émergente, pragmaticalisée; d'autre part il s'agit de relativiser la position prioritaire qu'aurait la syntaxe dans la définition des TCUs : dans les cas qu'elles examinent, la syntaxe définit des unités que les autres dimensions - prosodique, pragmatique, gestuelle, notamment - relativisent en construisant plutôt des continuités. Autrement dit, la prosodie et le geste peuvent produire des trajectoires d'unités qui dépassent les délimitations syntaxiques.

${ }^{13}$ Cf. aussi Selting: "TCUs are context-sensitive inferences, and thus are absolutely contingent on the activities constituted by participants » $(2000: 487)$.

${ }^{14}$ Fox précise utilement que : «It is important to note here that the claim is not that turn-taking determines grammar, or that all grammatical practices have arisen in response to the needs of turn-taking. The claim is, rather, that certain facets of grammatical organization, especially regarding beginnings and endings, may be responsive to the fact that utterances occur in turns. And, as with all functional pressures, languages may show different forms in response to the same pressure, so the existence of different grammars across languages does not invalidate the claim that languages are shaped in part by the needs of turn-taking. » (2007:303).

${ }^{15}$ Le terme même de «non-verbal » est doublement contestable, dans la mesure où il laisse entendre a) que le « nonverbal » serait défini par contraste avec le « verbal », réduit à en être un reflet négatif; b) que le «non-verbal » serait doté d'une logique séparée du «verbal ». Ce terme ne permet donc de reconnaître ni les spécificités propres de ces ressources, ni leur lien fort avec les ressources linguistiques. 